\title{
Analysis of in-line check valve with respect to the pipeline dynamics
}

\author{
Roman Klas ${ }^{1, *}$, Vladimír Habán ${ }^{1}$, and Pavel Rudolf ${ }^{1}$ \\ ${ }^{1}$ Brno University of Technology, Faculty of Mechanical Engineering, Victor Kaplan Department of Fluids Engineering, Technická 2 , \\ 61669 Brno, Czech Republic
}

\begin{abstract}
The following study is focused on the flow analysis within the concentric in-line check valve. In-line check valve is part of the pipe and shut off the stream of fluid. In case that backflow occurs on the downstream side, the valve is fully or partially lifted and then abruptly closes. This effect brings undesirable pressure shocks in the entire system. The study therefore deals with the CFD analysis of steady and unsteady flow with respect to velocity and pressure field. Especially unsteady calculation mode reflects the influence of local inertial acceleration acting on the fluid and on the valve. Analysis is complemented by equation of motion of a valve which interacts with the fluid.
\end{abstract}

\section{Introduction}

It is well known that the field of industrial valves is very broad. The reason for this fact is that the valves are designed for different functions. In-line check valve is usually used as backflow damming element and is part of a complex piping system. Features of this system may be more important than static or dynamic characteristics of the in-line check valve itself. It is also possible to say that the static behavior of the valve is not usually problematic in terms of the entire facility. Nevertheless, it is of course necessary to know the static behavior of the valve, in order to assess e.g. size of hydraulic losses and other flow characteristics of the valve. Paradoxically, the simplicity of its design and the simplicity of its main function represent the biggest source of problems along with its automatic operation.

Usually, when dealing with the valves we get by with knowledge of opening pressures depending on the nominal size of the valve. Valve lift and shut off times are assumed to be very short. Any possible mixing of operating substances is small for this reason and the same should apply to the magnitudes of static pressure in the pipeline. However these assumptions are not always valid and automatic shutoff function of the valve is the source of adverse pressure shocks and backflow of the fluid reinforced by spring. Nevertheless, the pipeline operating properties, under which the valve is deployed, are decisive.

Therefore, the aim of the study is to examine the risk of the real operating conditions of the in-line valve including its basic features in the form of static characteristics.

\section{Analytical and numerical methods and description of the valve}

A one-dimensional approach in the form of differential equations of momentum and CFD simulations for twodimensional and three-dimensional model were used to describe the behavior of the in-line valve. Parameters used in the differential equation are based on characteristics of the valve itself and with regard to the prediction of hydraulic losses also on CFD simulation. Therefore there is mutual effect of the approaches, but it is impossible to determine the hydraulic resistance of the valve otherwise than by experiments or CFD simulations $[1,2]$.

Table 1. Basic design parameters and description of numerical model.

\begin{tabular}{|l|l|l|}
\hline & 2D & 3D \\
\hline Pipeline diameter & \multicolumn{2}{|c|}{ DN100 } \\
\hline $\begin{array}{l}\text { Number of } \\
\text { computational cells }\end{array}$ & 750000 & 9 millions \\
\hline $\begin{array}{l}\text { Turbulence model } \\
\text { and near wall } \\
\text { modeling }\end{array}$ & $\begin{array}{l}\text { realizable k }-\varepsilon \\
\text { non equilibrium wall function }\end{array}$ \\
\hline $\begin{array}{l}\text { Boundary conditions } \\
\text { Onlet: velocity inlet } \\
\text { Outlet: pressure outlet }\end{array}$ \\
\hline Calculation mode & $\begin{array}{l}\text { unsteady, sliding mesh } \\
\text { incompressible flow }\end{array}$ \\
\hline
\end{tabular}

Examined in-line valve is a relatively new device, in which the entire experimental tests will be performed in the near future. Presently it is only known that operation

* Corresponding author: klas@fme.vutbr.cz 
of the valve is problematic in terms of pressure pulses and the slight backflow.

Momentum equation (1), Navier-Stokes equation (2) and continuity equation (3) were employed to describe the movement of the valve poppet in a one-dimensional approach.

Fig. 1 captures a basic view of the valve, which is identical with a 3D CFD model. Simplified 2D model is shown in Fig. 2. The main parameters of the valve and CFD simulations are recorded in Tab. 1. Possibilities for choice of an appropriate turbulence model can be found in [3-6] and the influence of the number of computational cells on the results of CFD simulations in [7] as well.

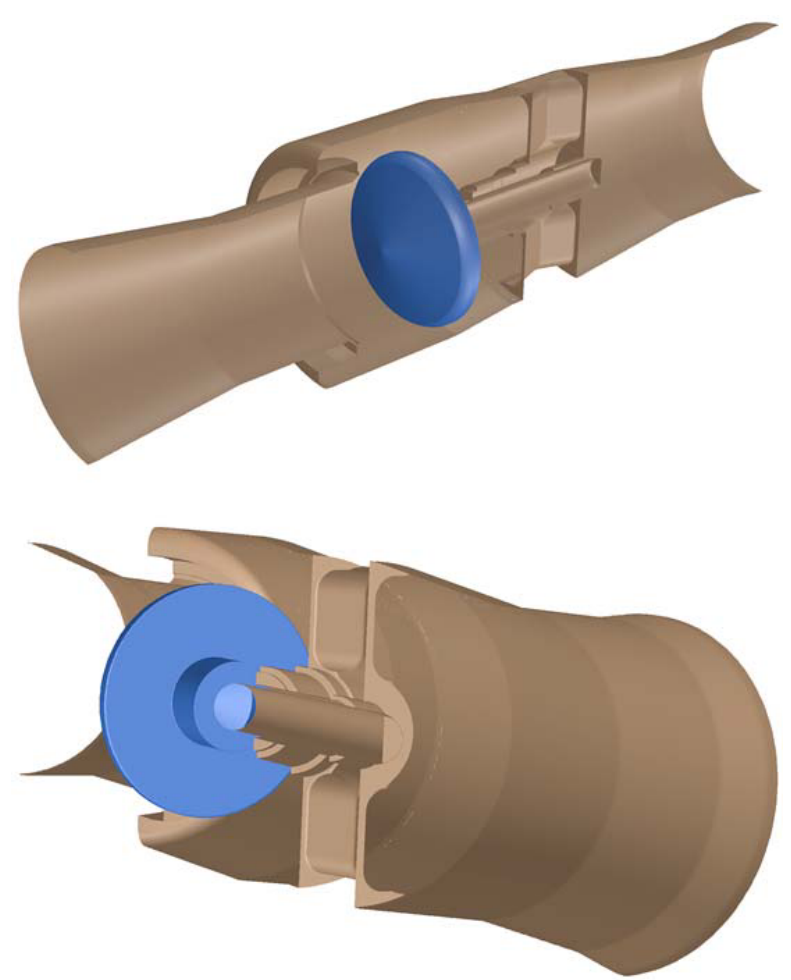

Fig. 1. The in-line valve, 3D simulation.

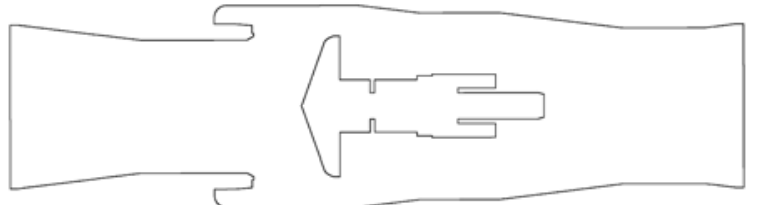

Fig. 2. The in-line valve, 2D simulation.

\section{Equation of motion}

One-dimensional solution of the basic momentum equation may not bring satisfactory results in many technical systems. However, the analytical solution of this equation provides the fundamental possibility of analyzing and understanding the phenomena and factors affecting the movement of poppet in liquid. Unfortunately, the complexity of determining of lift or valve poppet velocity as function of time is often so great that analytical solution is not always possible. Then it is necessary to rely on numerical solution of momentum equation.
Fig. 3 shows sketch of in -line valve in terms of the used symbols. The meaning all of the remaining used symbols is summarized in Tab.2.

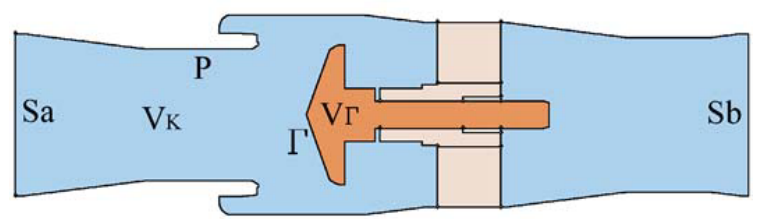

Fig. 3. Sketch of the in-line valve.

Table 2. List of symbols.

\begin{tabular}{|c|l|}
\hline $\mathrm{m}_{\Gamma}, \rho_{\Gamma}, \mathrm{V}_{\Gamma}$ & $\begin{array}{l}\text { mass, density and volume of valve } \\
\text { poppet }\end{array}$ \\
\hline $\mathrm{m}_{\mathrm{K}}, \rho_{\mathrm{K}}, \mathrm{V}_{\mathrm{K}}$ & mass, density and volume of liquid \\
\hline $\mathrm{b}_{\Gamma}$ & damping \\
\hline $\mathrm{k}$ & stiffness of the spring \\
\hline $\mathrm{x}_{0}$ & minimum spring preload \\
\hline $\mathrm{Q}, \mathrm{Q}_{\mathrm{m}}$ & volumetric and mass flow rate \\
\hline $\mathrm{Sa}, \mathrm{Sb}, \mathrm{S}$ & $\begin{array}{l}\text { areas of the input and output } \\
\text { surfaces of the valve }\end{array}$ \\
\hline $\mathrm{P}$ & solid wall of the valve \\
\hline$\Gamma$ & moving wall of the poppet \\
\hline $\mathrm{x}_{\mathrm{aCG}}$ & $\begin{array}{l}\text { Sa surface center of gravity } \\
\text { coordinates }\end{array}$ \\
\hline $\mathrm{x}_{\mathrm{bCG}}$ & $\begin{array}{l}\text { Sb surface center of gravity } \\
\text { coordinates }\end{array}$ \\
\hline $\mathrm{R}_{\mathrm{ab}}$ & $\begin{array}{l}\text { Hydraulic resistance of the valve } \\
\text { between the surfaces Sa and Sb }\end{array}$ \\
\hline
\end{tabular}

Momentum equation:

$$
m_{\Gamma} x_{i}+b_{\Gamma} \dot{x}_{i}+k\left(x_{i}+x_{0}\right)+F_{K}=G_{T i} .
$$

Integration of Navier-Stokes equation:

$$
\begin{aligned}
& \iiint_{V_{K}} \rho_{K}\left(\frac{\partial v_{i}}{\partial t}+\frac{\partial v_{i}}{\partial x_{j}} v_{j}\right) d V_{K}= \\
& \iiint_{V_{K}} \rho_{K} g_{i} d V_{K}-\iiint_{V_{K}} \frac{\partial p}{\partial x_{i}} d V_{K}+\iiint_{V_{K}} \frac{\partial \tau_{j i}}{\partial x_{j}} d V_{K} .
\end{aligned}
$$

Continuity equation:

$$
\frac{\partial v_{j}}{\partial x_{j}}=0
$$

The final form of equation of motion:

$$
\begin{aligned}
& \left(\rho_{\Gamma}+\rho_{K}\right) V_{\Gamma} \ddot{x}_{3}+b_{\Gamma} \dot{x}_{3}+k\left(x_{3}+x_{0}\right)= \\
& \rho_{K}\left(x_{b C G 3}-x_{a C G 3}\right) \frac{\partial Q}{\partial t}-R_{a b} S Q|Q| .
\end{aligned}
$$


The possibility how to determine $\mathrm{b}_{\Gamma}$ or formulate the momentum equation is indicated here e.g. [8]. It is also necessary to consider the effects of possible deformations of the poppet rigid body and its seat in some cases [9, 10]. However deformation of the rigid body is not included in this study.

\section{Static characteristics}

The first of the characteristics describing the essential behavior of the valve in terms of its operational properties are those, which describe behavior of coefficients $\mathrm{Kv}$ for fully open valve. Data obtained from 2D and 3D CFD simulations are shown for comparison in Fig. 4 and Fig.5. Determination of the correct magnitude of the flow coefficient depends of course on the position of the considered boundary surfaces, which are assigned to the valve. They were chosen as surfaces defining inlet and outlet of the valve, i.e. Sa and $\mathrm{Sb}$. See Tab. 2 and Fig. 3. Furthermore, the data corresponding to the surfaces on which the boundary conditions were located in CFD simulations, and which are situated at a distance of fifteen diameters of the pipe from the surfaces $\mathrm{Sa}$ and $\mathrm{Sb}$ are entered in the graphs.

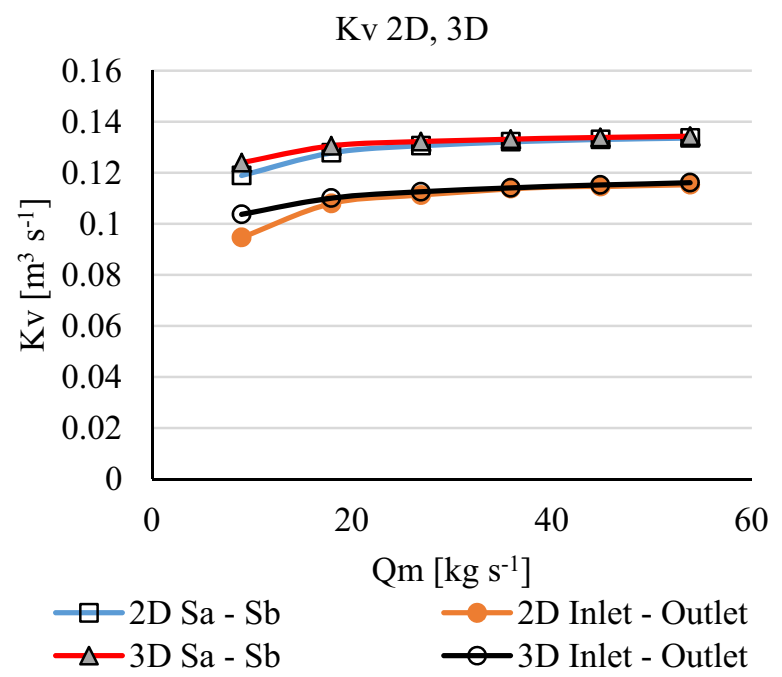

Fig. 4. Downstream flow coefficients.

It is apparent from Fig. 4 and Fig. 5 that the differences in $2 \mathrm{D}$ and $3 \mathrm{D}$ simulations in case of flow coefficients, especially at low mass flow rates, exist. . This effect is also evident in backflow, but obtained $\mathrm{Kv}$ values are almost constant.

The pressure drop in equation of motion is subsequently described by hydraulic resistance $\mathrm{R}_{\mathrm{ab}}$, see equations (4), and Tab. 2. The magnitude of the pressure drop is referenced to the same surfaces as in the case of flow coefficients Kv again, Fig. 6.

The axial forces depending on the static pressure difference are in both directions linear, as it was assumed, Fig. 7. Differences between 2D and 3D CFD simulations are greater due to the more complicated velocity and pressure fields around the valve poppet compared to the previous cases and other quantities.

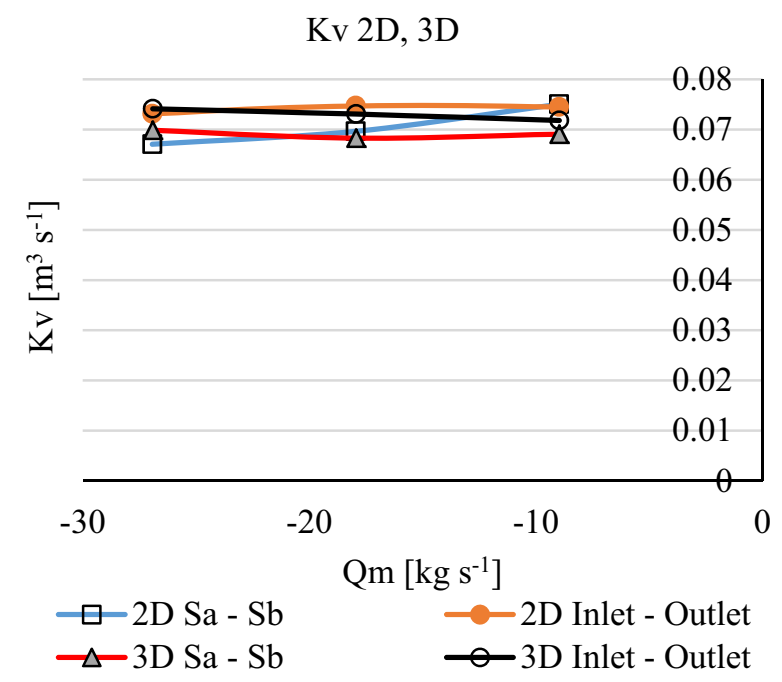

Fig. 5. Upstream flow coefficients.

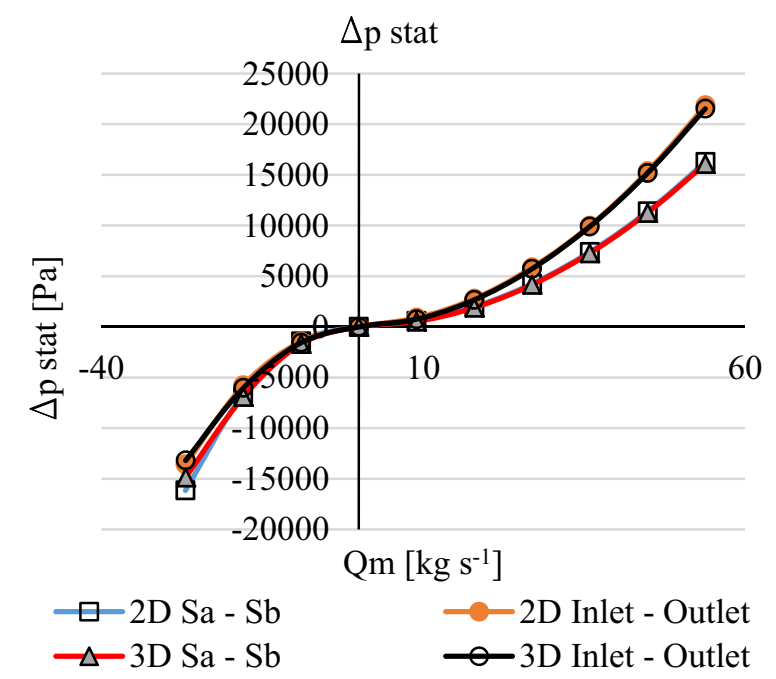

Fig. 6. Downstream and upstream static pressure differencies.

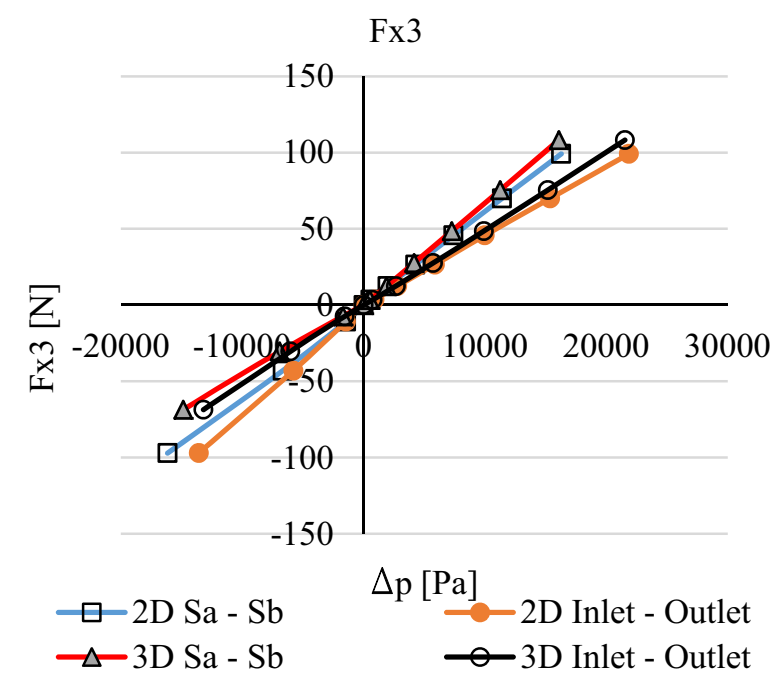

Fig. 7. The force magnitude along the axis of the valve acting on the poppet depending on the pressure drop.

The results are of course influenced by the static position of the poppet, which corresponds to a fully open valve, as already mentioned. It is also clear, with respect to the equation (4), that further study of valve properties 
requires a dynamic description of flow rate. Hitherto only a constant flow rate without the influence of timedependent flow rate was considered.

The above characteristics, depending on the mass flow rate, will therefore be part of the static characteristics. The flow rate will be defined by the following equation (5).

It is apparent from the equation (5) that the course of flow rate is linear and its derivative with respect to time corresponds to constant value. Valve shutoff time Ts $=$ $0.2 \mathrm{~s}$ is very short and for the current system is practically unrealistic. Shorter time changes were chosen primarily with respect to the CFD simulations, because a long time Ts would be very complicating as it will be explained later.

The following Fig. 8 represents an analogue of Fig. 6, which shows the pressure difference during the dynamic flow rate changes. The agreement of $2 \mathrm{D}$ and $3 \mathrm{D}$ CFD simulations is for the given scale almost absolute. It follows from the equation (4), that the offset of static pressure difference values should be constant with respect to the equation (5) compared to pressure difference in Fig. 6. Offset of curves $\mathrm{Sa}-\mathrm{Sb}$ and Inlet Outlet is given, of course, by their different distances from the valve poppet and different magnitudes of hydraulic losses in the piping outside the valve itself.

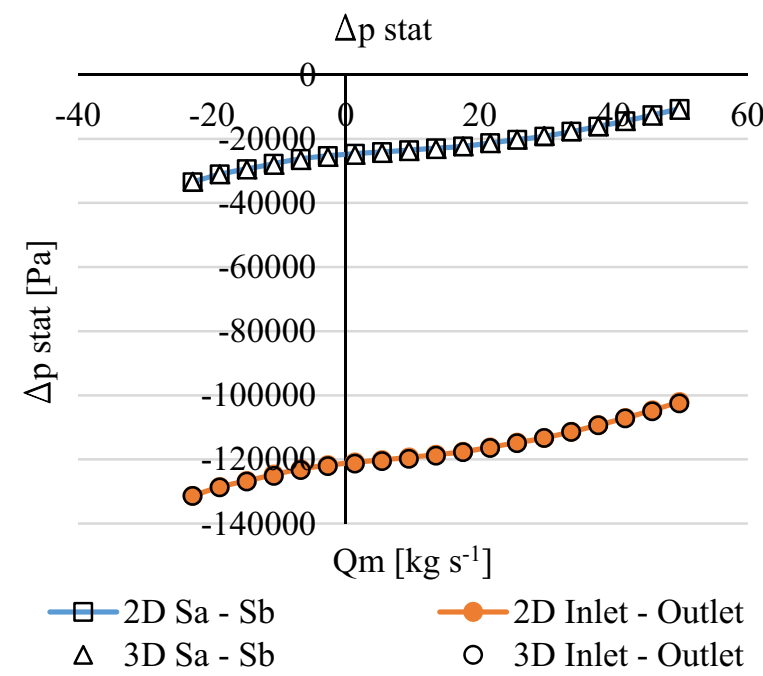

Fig. 8. Downstream and upstream static pressure difference.

It is apparent from a comparison of static pressure difference with and without considering time-dependent flow rate in Fig. 9, that there is a constant offset of the curves particularly in the area of positive mass flow rate values. This is in agreement with equations $(4,5)$. The differences are caused by different magnitudes of hydraulic losses, which result from different flow field around the poppet.

The magnitude of the force acting on the poppet depending on the static pressure difference is again approximately linear, Fig. 10.

It will be interesting to determine the magnitude of force acting on the valve poppet (Fig.11) in this context, if the poppet velocity and flow rate are prescribed according to equation (5). The magnitude of poppet force is obtained only from 2D CFD simulation.
Velocity of poppet is equal to $0.2 \mathrm{~ms}^{-1}$. Maximum distance of valve poppet motion corresponds to $\mathrm{x} 3 \max$ in Tab.1.

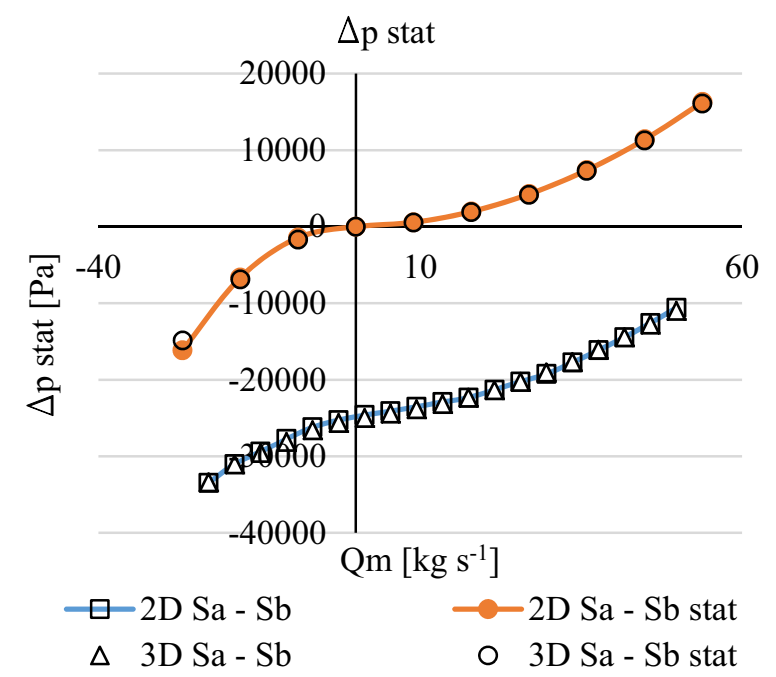

Fig. 9. Static pressure difference with and without timedependent mass flow rate.

Fx3

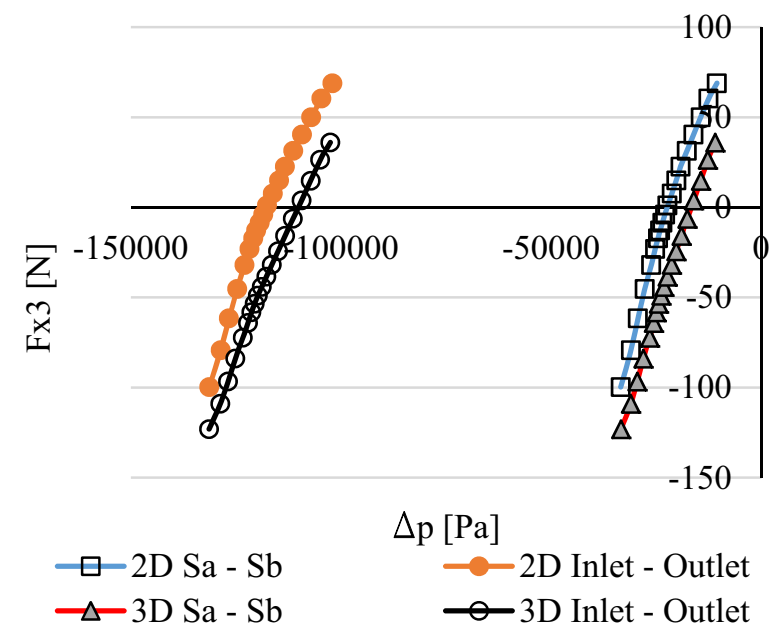

Fig. 10. The forces acting on the valve poppet.

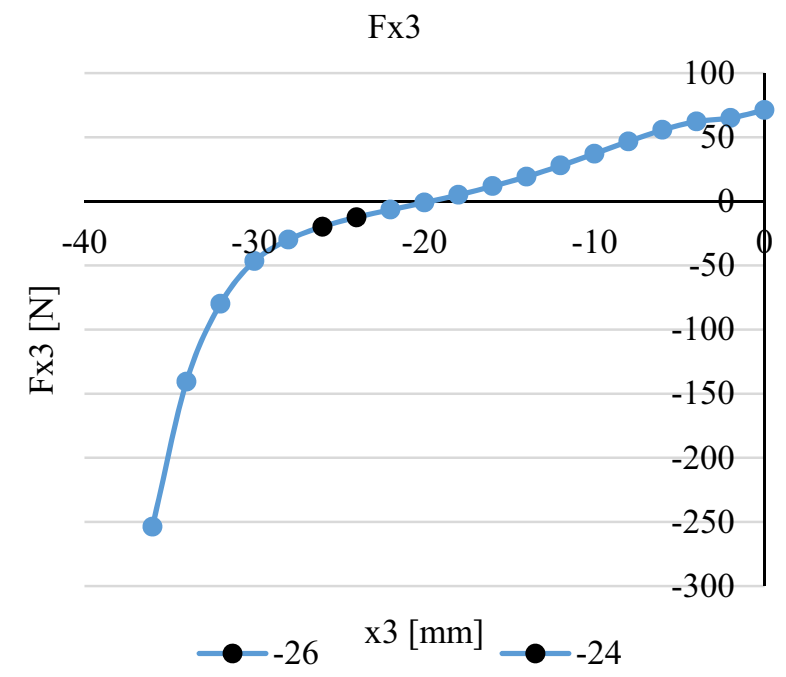

Fig. 11. The magnitude of forces acting on the valve poppet, depending on its position. 
The marked values correspond to points in Fig. 11, between which a change occurs in the direction of flow. The positive direction of all parameters corresponds to the positive direction of flow. Changing the magnitudes of the force is up to these values approximately linear, then the hydrodynamic forces acting on poppet start to dominate.

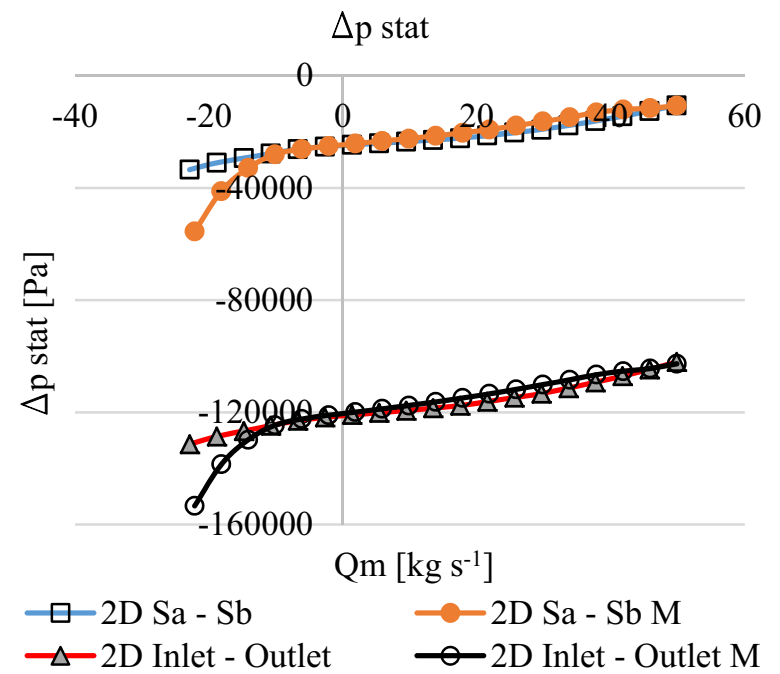

Fig. 12. Static pressure differences corresponding to the change of flow direction and movement of the valve poppet.

Fig. 12 depicts the change in static pressure between the surfaces $\mathrm{Sa}$ - Sb and Inlet - Outlet. The symbol "M" indicates data that were obtained in case of simultaneous changes of flow rate and poppet motion. It arises from Fig. 12 that the static pressure differences in $2 \mathrm{D}$ and $3 \mathrm{D}$ simulations correspond to each other up to the moment when the valve poppet is too close to the seat, and especially when a change of flow direction occurs. It follows that the poppet motion has for a certain operating range almost no influence on the pipeline system parameters. This was foreseen in introduction to the study and the data support this fact.

However, these findings also complicate the study of the poppet motion, because its behavior will largely depend on the dynamics of the whole pipeline.

\section{Dynamic characteristics}

Equation (4) was used to obtain the dynamic characteristics. Inclusion of following curves has a practical significance at this point, because to find solution of equation (4) it is necessary to know the resistance $R_{a b}$ or magnitude of the static pressure difference. Therefore, the results in the previous section will be used to determine the pressure difference. Complicated question is how to formulate a flow rate through the system.

Several test configurations have been chosen to describe the basic cases, which could occur during valve operation. The advantage is the possibility of a relatively quick and easy analysis of the poppet motion.

The simplest and well-known way of solving equation (4) is the case where the flow rate is constant, see Fig. 13.

$$
\mathrm{Q}_{0 \text { constant }}=0.04496011786 \mathrm{~m}^{3} \mathrm{~s}^{-1}
$$

Complications may arise in the formulation of resistance $R_{a b}$. It is possible to define the position of the poppet $\mathrm{x} 3$ in case of constant flow rate, and equation (4) also allows to check magnitude of damping $b_{\Gamma}$. We would obviously arrive to the same state, if we examined the case where the poppet is stationery and magnitude of flow rate corresponding to a specific position $\mathrm{x} 3$ would be unknown. The difference, of course, occurs with respect to the choice of initial conditions that define the transient state.

Another two cases defining the magnitude of the flow rate through the valve will now be closer to real conditions. The flow rate will be described by the equations (5) and (6). The curves in Fig. 13 labeled as cosine Qo1 and cosine Qo2 correspond to the equation (6). The only difference is in the initial flow rate Qo2. Graphical interpretation of the volume flow rate $(5,6)$ is entered in Fig. 13. The most controversial is the shutoff time of the valve, which was due to CFD simulations reduced ten times. However, there will be first presented results that correspond to the real time, Ts $=2 \mathrm{~s}$, and subsequently to a shorter shutoff time of the valve, Ts $=$ $0.2 \mathrm{~s}$.

$$
\begin{gathered}
Q=Q_{0}-\frac{Q_{0}-Q_{Z}}{T_{S}} t \\
Q=\frac{1}{2}\left(Q_{0}+Q_{Z}\right)+\frac{1}{2}\left(Q_{0}-Q_{Z}\right) \cos \left(\frac{\pi}{T_{Z}} t\right)
\end{gathered}
$$

Flow rate (6) corresponds best to the real system, in which the flow can be described by the following function (7) in the simplest cases.

$$
Q=Q_{0} e^{\alpha t} \cos (\beta t)
$$

Function (7) will be replaced just by (6) for basic characterization of flow rate.

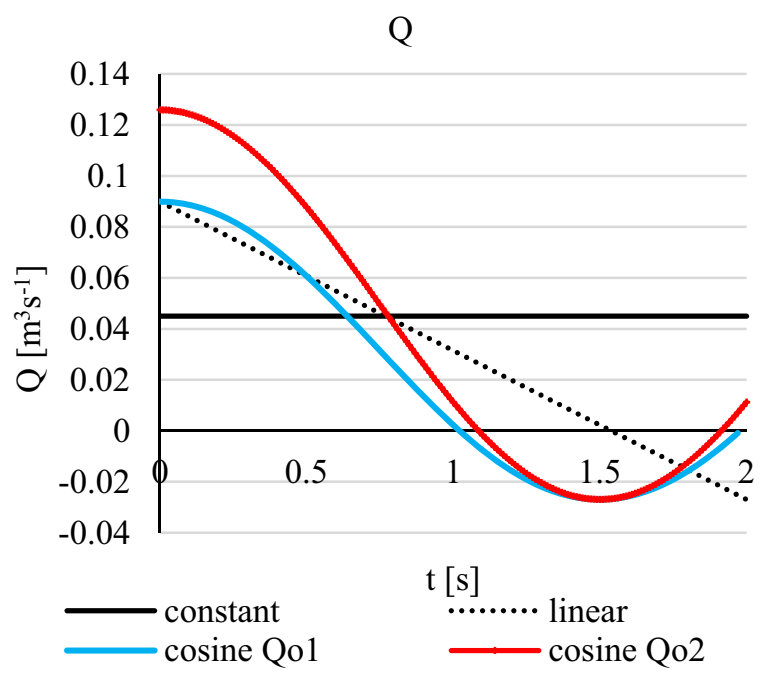

Fig. 13. Volume flow rates.

The constant flow rate is naturally chosen different from the one corresponding to fully open valve. Individual volume flow rates in Fig. 13 correspond to the 
time dependences of the poppet position $\mathrm{x} 3$ in Fig. 14 and the poppet velocity v3 in Fig. 15.

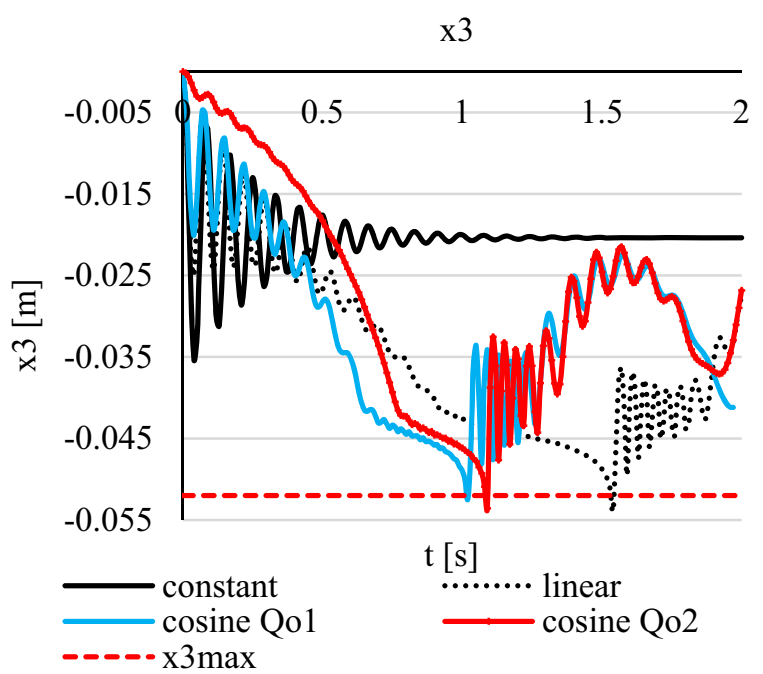

Fig. 14. Poppet position dependence on time and volume flow rate.

In Fig. 14, line $x 3$ max indicates the position of the poppet, which corresponds to the closed valve. If this value $\mathrm{x} 3$ is achieved and the direction of flow is positive, the backflow does not occur. Another part of the momentum equation has no importance in this case. The different maximum positions of cosine Qo1 and linear curves in Fig. 14, of course, correspond to the different points in which the flow rate reaches zero value, see Fig. 13.

v3

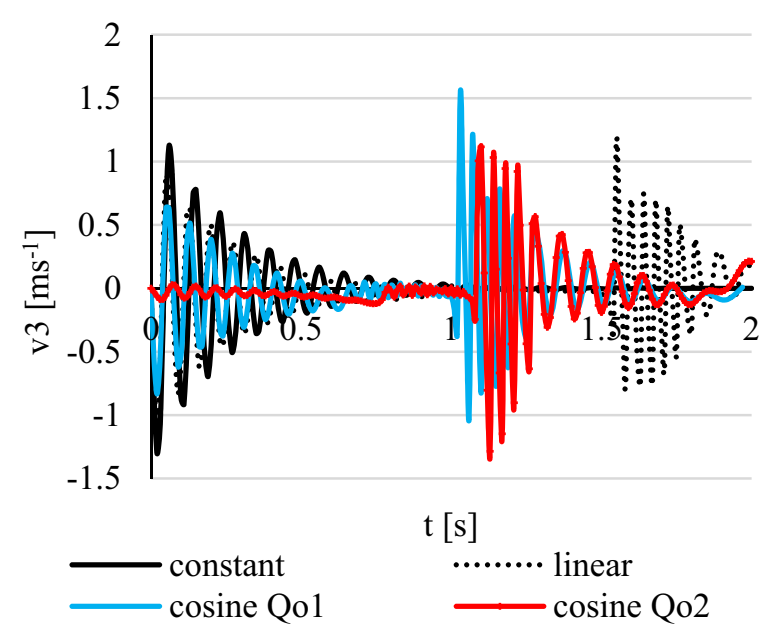

Fig. 15. Poppet velocity dependence on time and volume flow rate.

In Fig. 14 and 15 there are plotted data for the volume flow rates cosine Qo1 and cosine Qo2, which differ in magnitude of the initial flow rate. The influence of this fact on the $\mathrm{x} 3$ curve damping and a significant velocity v3 reduction is obvious. This is also related to the magnitude of attained overpressure [11]. Influences of other parameters, such as stiffness of the spring or the bellows can be found in $[12,13]$.
The shift of maximum value $\mathrm{x} 3$ on timeline is for these curves given by slightly different values of time when the flow rate Q reaches its zero values, see Fig. 13. Still, the results do not have a physical substantiation once $\mathrm{x} 3$ reaches $\mathrm{x} 3 \max$.

Equation (4) can be also modified by prescribing the poppet motion by poppet distance $\mathrm{x} 3$ and then timedependent volume flow rate, Fig. 16, can be determined. Description of poppet position $\mathrm{x} 3$ obviously corresponds to a constant poppet velocity.

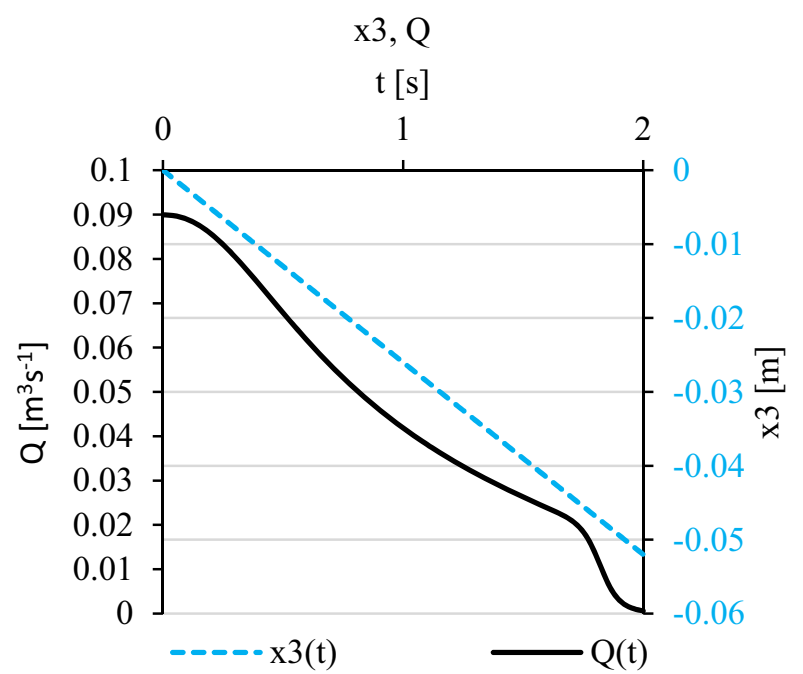

Fig. 16. The volume flow rate for a prescribed poppet position $\mathrm{x} 3$.

As it has already mentioned, CFD simulations were performed for the valve shutoff time $\mathrm{Ts}=0.2 \mathrm{~s}$ and for linearly changing volume flow rate Q in Fig. 13. Fig. 17 and Fig. 18 introduce position and velocity of the poppet for given shutoff time Ts of the valve.

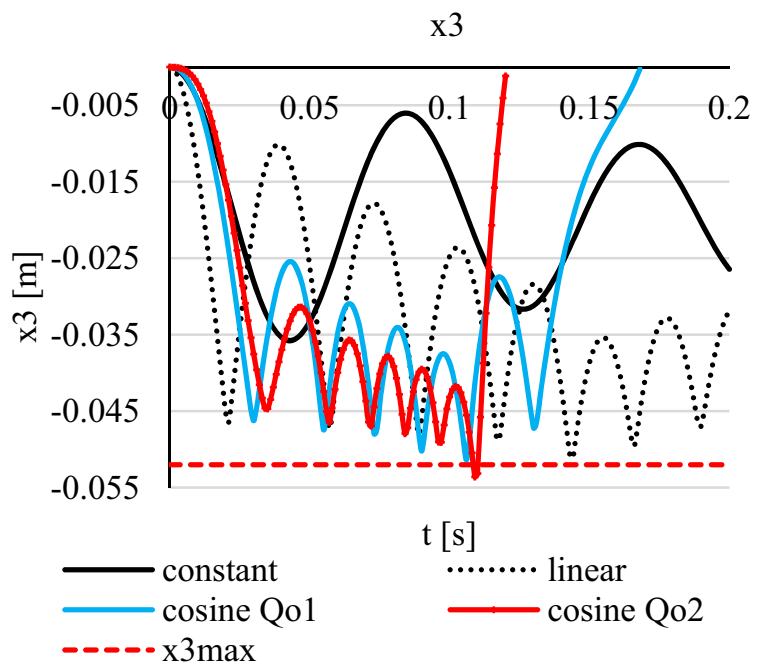

Fig. 17. The poppet position, shutoff time $T s=0.2 \mathrm{~s}$.

Data are not available for the flow cosine Qo1 and cosine Qo2 (Fig. 17, 18) in entirety full range, because numerical solution of momentum equations does not exist. The reason is partly evident also from the following equation (8), which is obtained from the original equation (4). 
The form of equation (4) can be described as a standard in terms of mechanical vibrations. Damping is now in less common form. Relationship of damping to vibration is also described in [14].

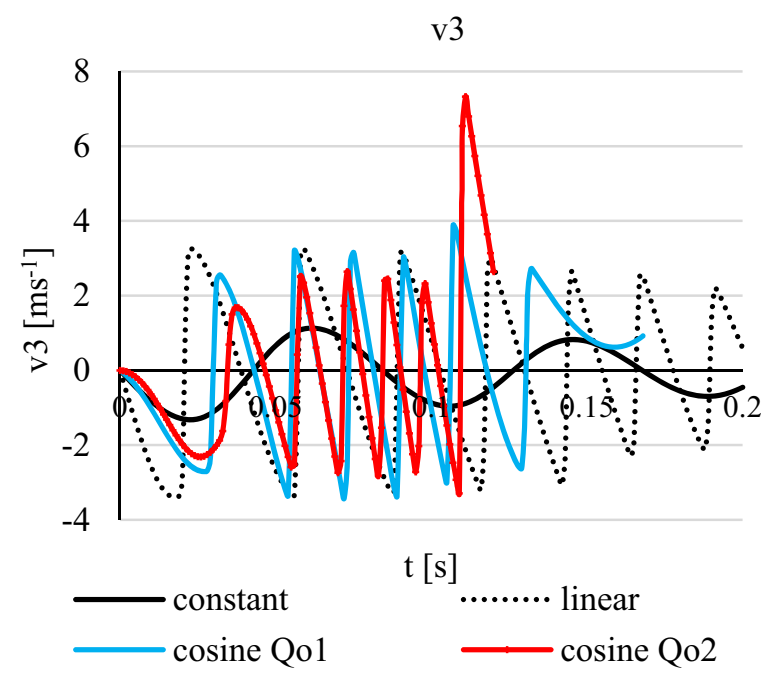

Fig. 18. The poppet velocity, shutoff time $\mathrm{Ts}=0.2 \mathrm{~s}$.

$$
\begin{gathered}
\ddot{x}_{3}+\frac{\left(b_{\Gamma}-\rho_{K}\left(x_{b C G 3}-x_{a C G 3}\right) \frac{\partial Q}{\partial x_{3}}\right)}{\left(\rho_{\Gamma}+\rho_{K}\right) V_{\Gamma}} \dot{x}_{3}+ \\
\frac{k}{\left(\rho_{\Gamma}+\rho_{K}\right) V_{\Gamma}} x_{3}=\frac{-R_{a b} S Q|Q|-k x_{0}}{\left(\rho_{\Gamma}+\rho_{K}\right) V_{\Gamma}}
\end{gathered}
$$

Damping in equation (8) will, to large extent,depend on the nature of changes in the flow rate with respect to coordinate $\mathrm{x} 3$ because $\rho_{\mathrm{K}}\left(\mathrm{x}_{\mathrm{bCGG} 3}-\mathrm{x}_{\mathrm{aCG} 3}\right)$ is always positive. This change will be zero for a constant flow rate and control function will only be function of $b_{\Gamma}$, which depends on the viscosity of liquid and on the shape of valve inner surfaces. We can estimate from Fig. 13 and functions cosine Qo1 and cosine Qo2 that the derivative of volume flow rate with respect to coordinate $\mathrm{x} 3$ is positive in certain moments. This fact may indicate negative damping, which reveals itself in Fig. 14 and Fig. 17 for curves labeled cosine Qo1 and cosine Qo2 at times approaching Ts.

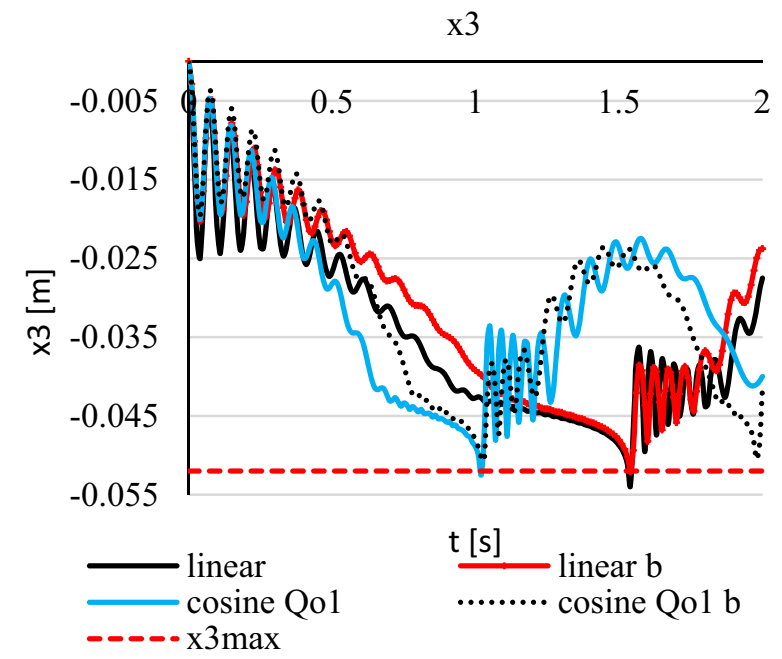

Fig. 19. The influence of damping on the poppet lift.

The general solution of equation (8) is well known and therefore will not be shown. Presence of flowing liquid, however, is the difference compared to the standard solution. Fig. 19 and Fig. 20 offer a comparison how the additional fluid damping effects the poppet motion. Variants in which only damping $b_{\Gamma}$ in equation (8) is considered are marked with the symbol "b".

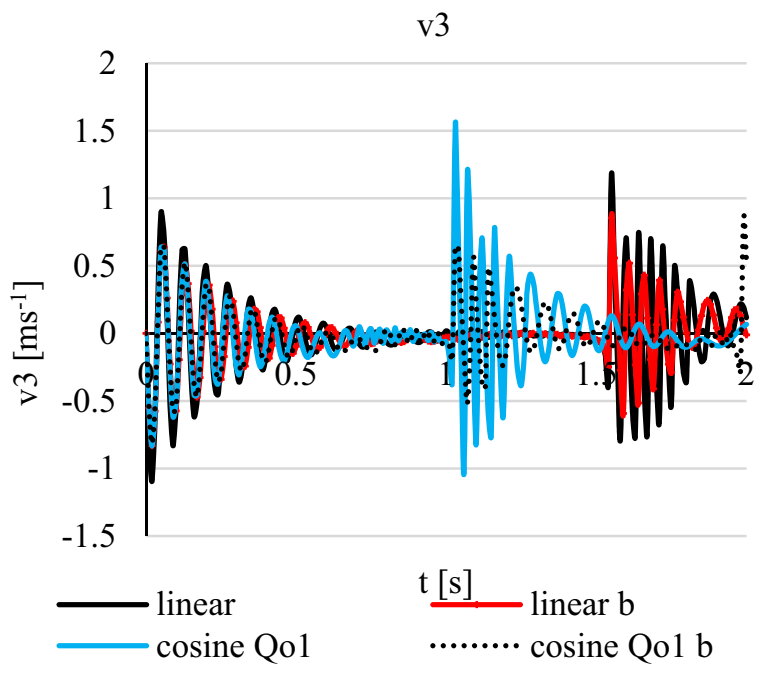

Fig. 20. The influence of damping on the poppet velocity.

It is apparent from Fig. 19 and Fig. 20 that the unsteady flow has rather a negative effect, because the vibration amplitudes are bigger. 
x3

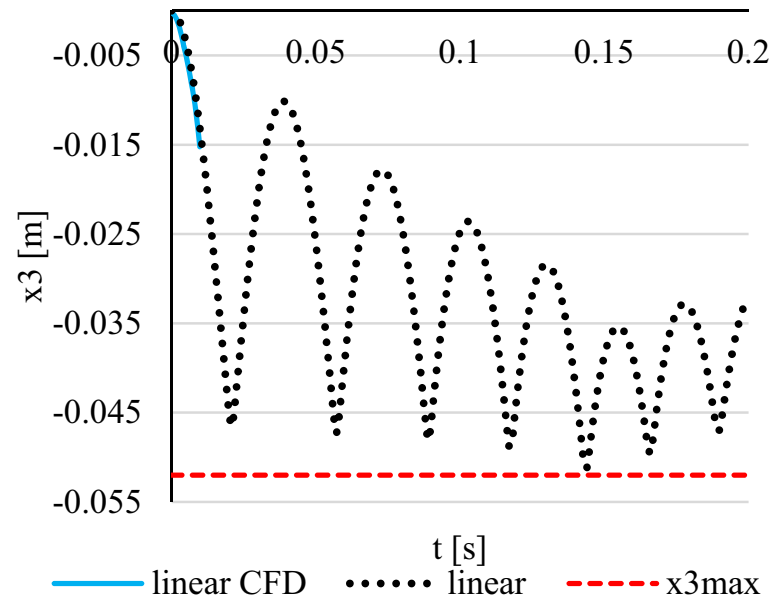

Fig. 21. The poppet lift comparison.

An attempt was also made to compare the results of time-dependent poppet lift with data from the 2D CFD simulation with one degree of freedom. The valve poppet moves by force resulting from the surrounding pressure field and the magnitude of its lift is recorded in time. One degree of freedom of the poppet is sufficient in this case, because the poppet moves only along the axis of the valve. However, the difficulty of this approach is with regard to the convergence of flow parameters and grid adaptation due to the great changes of poppet velocity and poppet lift. Therefore this type of simulation is unfeasible in full extent. Also, the influence of the spring is problematic because the spring complicates the code of UDF which defines the force acting on the poppet. The difficulty is not in the form of mathematical notation, but in the formulation of the spring force depending on the position itself. This spring force formulation causes a greater susceptibility of computational algorithm to instability and leads to disruption of the computing cycle. More can be found in [15].

From these reasons significantly shorter shutoff time of the valve was chosen, than are usual operating conditions. Of course, equation (4) quite simply allows this approach and for comparison is such approach beneficial. Fig. 21 therefore at least shows partial poppet lift comparison obtained from equation (4) and 2D CFD $1 \mathrm{DOF}$ simulation. The flow rate corresponds to the equation (5).

It seems there is only few CFD simulation data at first sight, but it is extremely time-consuming to obtain this data due to very short computational time step. However, the resulting consensus is at least in a small section of poppet lift very good. Relatively long total poppet lift $\times 3$ max from fully open to closed valve is in this case problematic.

\section{The velocity and pressure field}

It will be important to find out how much the velocity and pressure fields are different due to differences between $2 \mathrm{D}$ and $3 \mathrm{D}$ CFD simulations. The fin is the most fundamental difference between both simulations, see Fig. 1. The housing of the valve poppet is attached just to the fin. The fin is obviously not placed in 2D simulations. The fin thickness is not too large, therefore assumption of flow axisymmetry in the rest of the valve domain might be plausible.
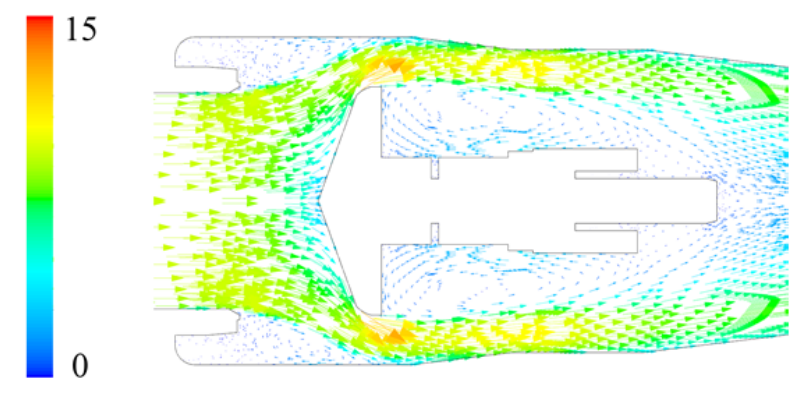

Fig. 22. Absolute velocity field obtained from 2D CFD simulation, flow rate Q1.
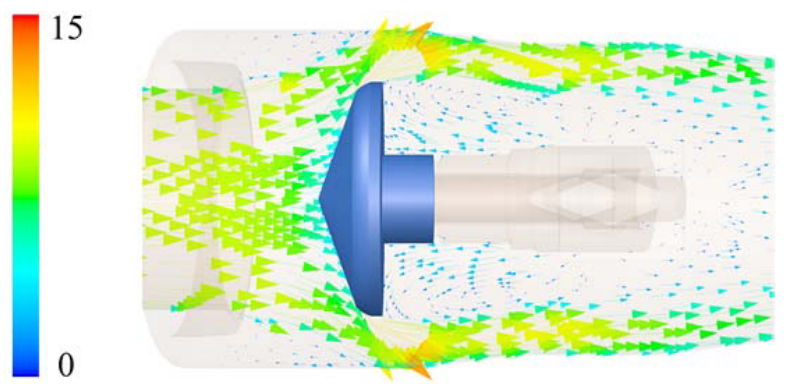

Fig. 23. Absolute velocity field obtained from 3D CFD simulation, flow rate Q1.

Cross-section outside of the fin of 3D valve body is depicted Fig. 22 - 25 to verify this fact. Figs. Fig. 22 - 25 show a comparison of velocity and pressure fields for the flow rates Q1 $=0.053913636 \mathrm{~m}^{3} \mathrm{~s}^{-1}$ and Fig. $26-29$ for flow $\mathrm{Q} 2=-0.026956818 \mathrm{~m}^{3} \mathrm{~s}^{-1}$.

2D CFD simulation of the valve can be examined in more detail with respect to the discretization and solution methods here [16].

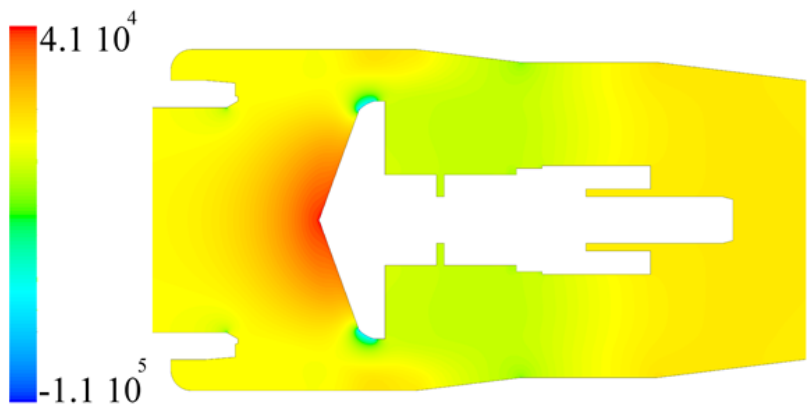

Fig. 24. Static pressure field obtained from 2D CFD simulation, flow rate Q1. 


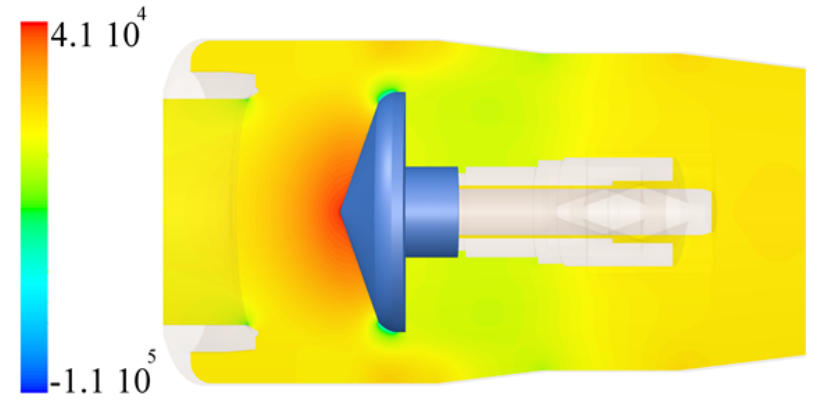

Fig. 25. Static pressure field obtained from 3D CFD simulation, flow rate Q1.
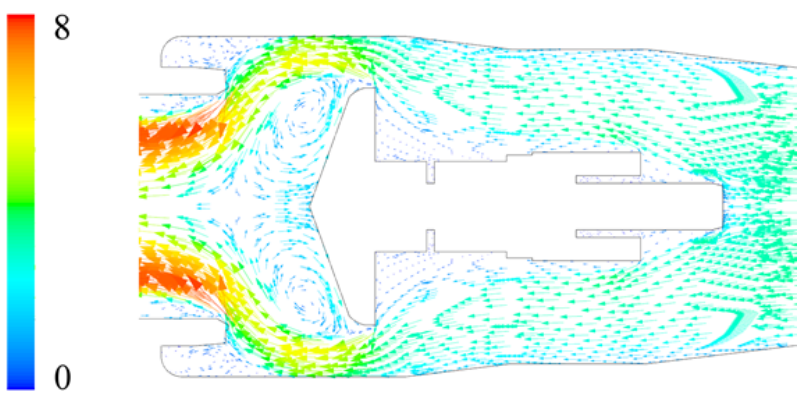

Fig. 26. Absolute velocity field obtained from 2D CFD simulation, flow rate $\mathrm{Q} 2$.
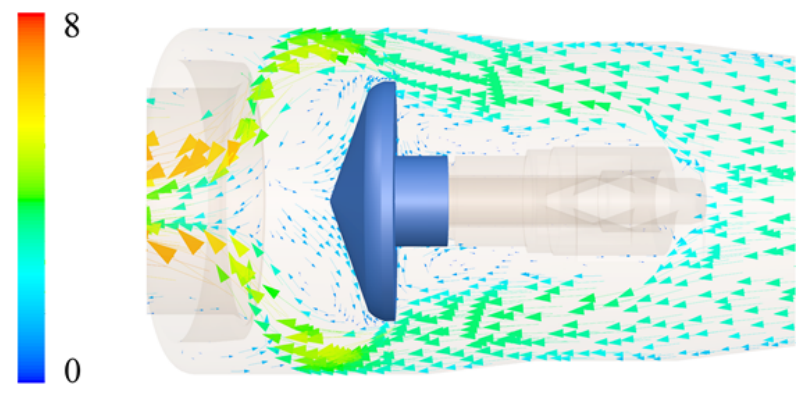

Fig. 27. Absolute velocity field obtained from 3D CFD simulation, flow rate Q2.

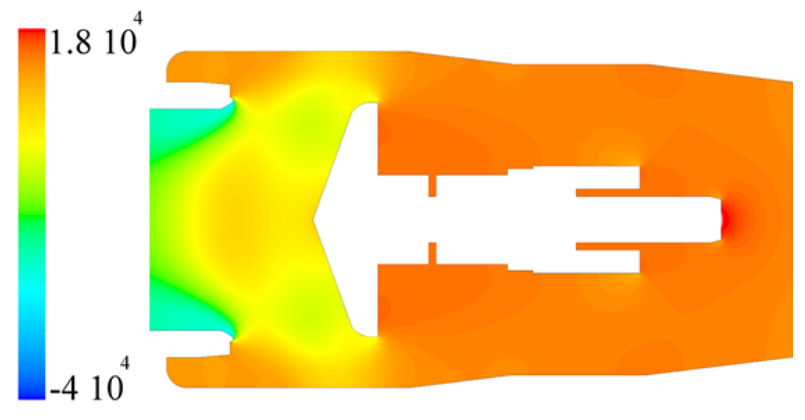

Fig. 28. Static pressure field obtained from 2D CFD simulation, flow rate Q2.

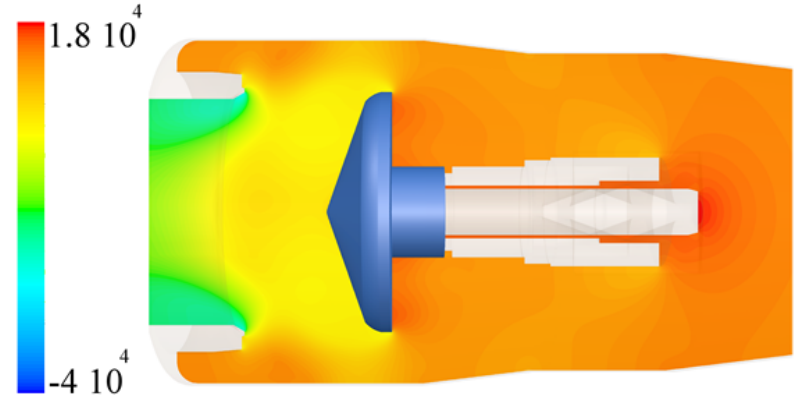

Fig. 29. Static pressure field obtained from 3D CFD simulation, flow rate Q2.

It is obvious that the agreement is very good. The same scales of static pressure and absolute velocity were chosen for this purpose. Similar results are also obtained for the other flow rate.

Note: It is necessary to monitor the flow around the poppet also in terms of water erosion and cavitation, [17].

\section{Conclusions}

Experiment, which has only partially been carried out so far, will decide about validity of presented data and characteristic curves. . It is important to assess at least the basic correspondence between equation of motion of valve poppet and CFD simulations of two-dimensional and three-dimensional models. Hydraulic resistance determination is also a problematic point, which can be defined only by experiment or simulation CFD.

From the analyzed alternatives $3 \mathrm{D}$ simulation should provide the most accurate results. It was therefore important to establish a comparison between 2D and 3D model in order to obtain some parameters from the simpler 2D model. Very good agreement was shown on the attached characteristics between $2 \mathrm{D}$ and $3 \mathrm{D}$ models. The same can be mentioned also for the comparison of the velocity and pressure fields.

The operating characteristics and force acting on valve poppet are essential in terms of valve properties. It can be stated about the force characteristic, that its dependence on the pressure difference remains linear as in the case of stationary flow.

From the case of prescribed poppet motion it can be concluded that poppet motion has no significant influence on the flow field except the region where hydrodynamic force considerably increases. The reason is that the poppet lift is relatively long.

Correct assessment of the unsteady flow influence is also essential in the formulation of the basic equations of motion in terms of its practical usability. Verification of influence of unsteady flow in CFD simulations revealed that the described simplified model is quite useful in the equation of motion and corresponds to the results of CFD simulations.

Effect of transient flow also causes a significant decrease in static pressure, which brings problems with cavitation. Cavitation mode was not simulated, but may obviously have an important influence on the dynamic 
properties of the valve. Cavitation can be simulated in later studies.

The dynamic lifts and velocities are the most important characteristics of the poppet, which of course greatly depend on the flow rate through the valve. The actual flow rate depends on the characteristics of the whole pipeline and the flow rate cannot be restricted to valve itself Therefore only few operating states corresponding to the basic cases be simulated. Especially those the which occur in real operation or which may be interesting for analyzing of the poppet motion.

The influence of unsteady flow on the damping of poppet motion is evident from the attached characteristics. Its magnitude for various positions of the poppet can cause oscillations. Also conditions in which the backflow should occur were simulated. Though, the situation of fully closed valve never occurs on the plotted characteristics. However, it is necessary to note the effect of cavitation, which occurs according to the rate of pressure drop, and which may affect the dynamics and function of the valve.

This work has been supported by Technology Agency of the Czech Republic under the project Innovative research of check valves for extreme operating conditions in energetics TH01011352.

\section{References}

1. J. A. Davis, M. Stewart, J. Fluids Eng. 124, 3, pp. 772-777 (2002)

2. J. A. Davis, M. Stewart, J. Fluids Eng. 124, 3, pp. 778-783 (2002)

3. L. Davidson, An Introduction to Turbulence Models 97/2, (2016)

4. A. Oza, S. Gosh, K. Chowdhury, CFD Modeling of Globe Valves for Oxygen Application, 1356-1363 (16th Australasian Fluid Mechanics Conference, 2007)

5. S. Sibilla, M. Gallati, J. Fluids Eng. 130, 121101 (2008)

6. V. Yakhot, S. A. Orszag, S. Thangam, T. B. Gatski, C. G. Speziale, Phys. Fluids A 4_7_, pp. 1510-1520 (1992)

7. A. Budziszewski, L. Thorén, CFD simulation of a safety relief valve for improvement of a onedimensional valve model in RELAP5 (Department of Applied Physics, 2012)

8. M. Domagala, Czasopismo Techniczne 3-M, pp. 2429 (2008)

9. S. W. Lee, D. Y. Shin, C. W. Byun, H. J. Yang, I.C. Paek, J. of Achievements in Mat. and Manufact Eng. 23, pp. 67-70 (2007)

10. X. J. Yuan, K. H. Guo, SCIENCE CHINA Technological Sciences 58, 6 (2015)

11. X. G. Song, J. H. Jung, H. S. Lee, D. K. Kim, Y. Ch. Park, 2-D Dynamic Analysis of a Pressure Relief Valve by CFD (WSEAS Conferences, Hangzhou, China, 2010)
12. X. Song, L. Cui, M. Cao, W. Cao, Y. Park, W. M. Dempster, Energy Conversion and Management 81, pp. 407-419 (2014)

13. S. Chabane, S. Plumejault, D. Pierrat, A. Couzinet, Vibration and chattering of conventional safety relief valve under built up back pressure (IAHR International Meeting, 2009)

14. A. Singh, Nuclear Eng. and Design 72, pp.197-204 (1982)

15. X. G. Song, L. T. Wang, Y. C. Park, W. Sun, Procedia Engineering 130, pp. 87-94 (2015)

16. A. Beune, Analysis of high-pressure safety valves, (Eindhoven University Press, 2009)

17. B. E. Dallstream, B. A. Fricke, B. R. Becker, Swing Check Valve Design Criteria and CFD Validation (ICONE14, 2006) 